\title{
UFT plus gemcitabine combination chemotherapy in patients with advanced non-small-cell lung cancer: a multi-institutional phase II
} trial

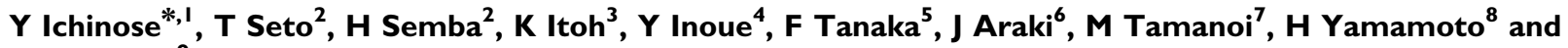 \\ N Iwamoto"
}

'Department of Thoracic Oncology, National Kyushu Cancer Center, 3-I-I, Notame, Minami-ku, Fukuoka 81 I-1 395, Japan; ${ }^{2}$ Division of Respiratory Diseases, Kumamoto Regional Medical Center, Kumamoto, Japan; ${ }^{3}$ Department of Respiratory Medicine, Shin Beppu Hospital, Oita, Japan; ${ }^{4}$ Department of Respiratory Medicine, Isahaya Insurance General Hospital, Nagasaki, Japan; ${ }^{5}$ Department of Respiratory Medicine, Kumamoto City Hospital, Kumamoto, Japan; ${ }^{6}$ Department of Respiratory Medicine, Yamaguchi Central Hospital, Yamaguchi, Japan; ${ }^{7}$ Department of Respiratory Medicine, Minamata General Medical Center, Kumamoto, Japan; ${ }^{8}$ Department of Respiratory Medicine, Asou lizuka Hospital, Fukuoka, Japan; ${ }^{9}$ Respiratory Organ and Diabetes Center, Saiseikai Kumamoto Hospital, Kumamoto, Japan

A multi-institutional phase II trial was conducted to evaluate the efficacy and toxicity of combination chemotherapy consisting of gemcitabine and UFT, which is composed of tegafur and uracil, for non-small-cell lung cancer (NSCLC) patients. Patients with advanced NSCLC received an oral administration of UFT (tegafur $200 \mathrm{mg} \mathrm{m}^{-2}$ ) b.i.d. from days I to I 4 and intravenous injection of gemcitabine $900 \mathrm{mg} \mathrm{m}^{-2}$ on days 8 and 15. This treatment was repeated every 4 weeks. A total of 44 patients were enrolled into this trial. The median age of all patients was 74 years, with 23 patients younger than 75 years and 21 patients with 75 years of age or older. A total of 18 patients (4I\%) achieved a partial response. The median survival time was 13.2 months and the |-year survival rate was $59 \%$. The most common grade 3-4 toxicity was neutropenia (57\%). The frequency of grade 3 nonhaematologic toxicities was less than $5 \%$. In addition, no significant difference in the response, survival or toxicities was observed between the patients younger than and those older than 75 years of age. This combination chemotherapy demonstrated a promising effectiveness and acceptable toxicity in patients with advanced NSCLC, even in patients older than 75 years.

British Journal of Cancer (2005) 93, 770-773. doi:I0.I038/sj.bjc.660278I www.bjcancer.com

Published online 20 September 2005

(C) 2005 Cancer Research UK

Keywords: uracil-tegafur; UFT; gemcitabine; NSCLC; elderly

UFT is an oral anticancer agent composed of tegafur and uracil at a $1: 4$ fixed molar ratio (Fujii et al, 1979). Although the clinical effectiveness of the single agent against advanced non-small-cell lung cancer (NSCLC) has not been evaluated in adequate sample size (Keicho et al, 1986), a recent randomised phase III trial in 984 patients with completely resected stage I adenocarcinoma demonstrated that postoperative adjuvant chemotherapy with UFT significantly prolonged the survival of patients in comparison to observation alone (Kato et al, 2004). The combination chemotherapy of UFT plus cisplatin has also been reported to be an effective treatment for advanced NCSLC. The combination chemotherapy consisting of a daily administration of UFT for 2 or 3 weeks and a bolus injection of cisplatin at the mid-cycle of the administration of UFT yields a response rate of $29-38 \%$, and a median survival time of 10-13 months (Ichinose et al, 1995, 2000; Saito et al, 2001).

Gemcitabine is an active anticancer agent for the treatment of NSCLC. The objective response rates of patients with advanced NCSLC treated with gemcitabine alone and the combination chemotherapy of platinum plus gemcitabine range from 20 to $26 \%$

*Correspondence: Dr Y Ichinose; E-mail: yichinos@nk-cc.go.jp Received 22 March 2005; revised 10 August 2005; accepted 10 August 2005; published online 20 September 2005 and from 25 to $61 \%$, respectively (Harper, 2003). A median survival time of 8-16 months in patients treated with the combination chemotherapy has been reported (Harper, 2003).

Both 5-FU generated from tegafur in UFT and gemcitabine are antimetabolites but inhibit DNA synthesis via a different pathway. The main mechanism of inhibiting DNA synthesis by $5-\mathrm{FU}$ is due to 5-FU-derived fluorodeoxy monophosphate binding to thymidylate synthase (Madajewicz et al, 1984). The incorporation of gemcitabine triphosphate, which is generated from the phosphorylation of gemcitabine by deoxycytidine kinase, into DNA is most likely the major mechanism by which gemcitabine exerts its cytotoxic action (Huang et al, 1991). Such different antitumour mechanisms suggest a potential synergism between 5-FU and gemcitabine. This potential synergism has been indeed observed in in vitro studies using various cancer cell lines (Schulz et al, 1998; Peters et al, 2000).

In our prior phase I trial, the combination chemotherapy of UFT plus gemcitabine was found to be feasible (Seto et al, 2002). The most appropriate schedule and dosing was $200 \mathrm{mg} \mathrm{m}^{-2}$ of UFT b.i.d for 14 consecutive days with $900 \mathrm{mg} \mathrm{m}^{-2}$ gemcitabine on days 8 and 15 . The main toxicity was haematologic. The overall response rate was $33 \%$, while the rate was $45 \%$ in the 13 patients without any prior chemotherapy. With these backgrounds, we conducted a phase II trial of combination chemotherapy using 
UFT plus gemcitabine. In the present multi-institutional phase II trial, we found the proportion of elderly, especially patients older than 75 years of age, to be high. Therefore, the results of all patients in the phase II trial and the differences between patients younger than 75 years and those 75 years of age or older were evaluated.

\section{MATERIALS AND METHODS}

\section{Patient eligibility}

The patients were eligible for this phase II trial if they had been either cytologically or histologically confirmed to have NSCLC; stage IIIB without any indications for radiotherapy or stage IV; measurable disease; no prior chemotherapy; an Eastern Cooperative Oncology Group (ECOG) performance status (PS) of 0,1 or 2; a projected life expectancy of at least 3 months. Other eligibility criteria regarding organ functions were as follows: a leucocyte count of $4000-12000 \mathrm{l}^{-1}$; platelet count of $100000 \mu \mathrm{l}^{-1}$ or greater; haemoglobin level of $9 \mathrm{~g} \mathrm{dl}^{-1}$ or greater; a serum bilirubin level less than $1.5 \mathrm{mg} \mathrm{dl}^{-1}$; serum aspartate aminotransferase and alanine aminotransferase levels of twice the upper limit or less; a serum creatinine level of $1.5 \mathrm{mg} \mathrm{dl}^{-1}$ or less. For staging, all patients underwent a computed tomography (CT) scan of the thorax, including the upper abdomen, and either brain CT or magnetic resonance images of the brain, and a radioisotopic bone scan were performed for almost all patients.

Any patients who were pregnant or had concomitant serious diseases, a concomitant malignancy, pleural effusion necessitating treatment or symptomatic cerebral involvement were excluded from the study. However, patients whose malignant pleural effusion was controlled by intrapleural hypotonic cisplatin treatment (Ichinose et al, 2003) were eligible. Written informed consent was required from all patients and the protocol was approved by the institutional ethics committee of each of the participating institutions. On entrance to the study, the eligibility of patients was checked via facsimile by the central administration office of the Kyushu Yamaguchi Thoracic Oncology Group (Fukuoka).

\section{Treatment schedule}

UFT (tegafur $400 \mathrm{mg} \mathrm{m}^{-2}$ day $^{-1}$ ) in the form of a 100 -mg capsule (100 mg of tegafur plus $224 \mathrm{mg}$ of uracil) was given orally in two separate doses, before meals, from days 1 to 14 . The dose was rounded up or down to the nearest $100 \mathrm{mg}$. Most patients received UFT three capsules (tegafur $300 \mathrm{mg}$ and uracil $672 \mathrm{mg}$ ) b.i.d. Gemcitabine $\left(900 \mathrm{mg} \mathrm{m}^{-2}\right.$ ) was dissolved in $20 \mathrm{ml}$ of physical saline and then diluted further with physical saline or $5 \%$ glucose to a volume of $250 \mathrm{ml}$. The gemcitabine solution was administered by intravenous drip infusion over $30 \mathrm{~min}$ on days 8 and 15 . On the day gemcitabine was administered, a complete blood count was performed and the drug was administered only when the leucocyte count was $2000 \mu \mathrm{l}^{-1}$ or higher and the platelet count was 70000 or higher $\mu \mathrm{l}^{-1}$. If these requirements were not met, then drug administration was postponed for a maximum of 4 days. The treatment regimen was repeated every 4 weeks and at least two cycles were administered unless disease progression or an unacceptable toxicity occurred. A leucocyte count of $3000 \mu \mathrm{l}^{-1}$ or greater and the entry eligibility criteria regarding organ functions had to be satisfied to start the next cycle. The doses of gemcitabine were reduced to $800 \mathrm{mg} \mathrm{m}^{-2}$ either when grade 4 haematologic toxicities occurred or when the administration of gemcitabine on day 15 was skipped in a prior cycle. The doses were increased to $1000 \mathrm{mg} \mathrm{m}^{-2}$ if both nadirs of the leucocyte and platelet counts were more than 3000 and $100000 \mu \mathrm{l}^{-1}$, respectively, in a prior cycle.

\section{Evaluation of response and toxicity}

All eligible patients who received any part of the treatment were considered assessable for response and toxicity. The complete blood count, and blood chemistry studies were repeated weekly. The response was assessed based on the chest X-ray or CT scan findings that initially had been used to define tumour extent. The response was evaluated according to the criteria of the World Health Organization (Miller et al, 1981). A central radiologic review was performed to determine the eligibility of patients and the response of treatment. Any adverse events were graded according to the National Cancer Institute-Common Toxicity Criteria (NCI-CTC) version 2.0.

\section{Statistical analysis}

The primary end point of this study was to determine the tumour response rate produced with this treatment protocol. Based on the assumption that a response rate of higher than $40 \%$ would warrant a further investigation of this combination chemotherapy, and that a rate of below $20 \%$ would make such an investigation unnecessary, a sample size of 36 patients was required with an alpha error of 0.05 and a beta error of 0.2 . Therefore, the accrual of 40 patients was planned for a 2 -year period since several ineligible patients might be identified in the course of the study.

The overall survival of the eligible patients was defined as the time from the start of the treatment until death from any cause and it was estimated by the Kaplan-Meier method. Differences between the proportions were evaluated by the chi-square test. The data were considered to be significant when the $P$-value was 0.05 or less.

\section{RESULTS}

\section{Patient characteristics}

From July 2000 to September 2002, 44 patients were entered into this phase II trial. Since no upper age limit for the eligibility criteria was established, in contrast to other Japanese trials, many elderly patients were included in this trial. The patient characteristics classified by an age of less than 75 years of age or 75 years of age and older are shown in Table 1 . There were no statistically significant differences in the proportions regarding gender, performance status, stage, histology or previous treatment between the two $(<75 v s \geqslant 75$ years) groups. There were 33 patients who were 70 years of age or older.

\section{Treatment delivery}

The median number of treatment cycles for all patients was three as shown in Table 2 . In all, $39(89 \%)$ patients received at least two cycles of the treatment. The reasons for terminating the chemotherapy before the second treatment cycle were adverse events in three patients, a progression of dementia in one and patient refusal in one. Although the proportions of the cycles administered between the two-age groups showed no significant difference, $10(48 \%)$ patients in the older age group received five or more cycles of the treatment.

The administration of gemcitabine on day 15 was skipped in $10(5 \%)$ of a total of 196 cycles. A dose decrease to $800 \mathrm{mg} \mathrm{m}^{-2}$ and the increase to $1000 \mathrm{mg} \mathrm{m}^{-2}$ were reported in four and five patients, respectively.

\section{Adverse events}

The main adverse events were haematologic toxicities as shown in Table 3. Grade 3 and 4 neutropenia was reported in $57 \%$ of the patients and grade 4 in $20 \%$, while no grade 4 anemia or 
Table I Patient characteristics

\begin{tabular}{|c|c|c|c|}
\hline & $\begin{array}{c}<75 \text { years } \\
(n=23)\end{array}$ & $\begin{array}{c}\geqslant 75 \text { years } \\
(n=21)\end{array}$ & $\begin{array}{c}\text { Total } \\
(n=44)\end{array}$ \\
\hline \multicolumn{4}{|l|}{ Age } \\
\hline Median (range) & $70(58-74)$ & $78(75-89)$ & $74(58-89)$ \\
\hline \multicolumn{4}{|l|}{ Gender } \\
\hline Male/female & $7 / 16$ & $11 / 10$ & $18 / 26$ \\
\hline \multicolumn{4}{|c|}{ Performance status (ECOG) } \\
\hline $0 / 1 / 2$ & $8 / 13 / 2$ & $8 / 13 / 0$ & $16 / 26 / 2$ \\
\hline \multicolumn{4}{|l|}{ Stage } \\
\hline III/IV & $5 / 18$ & $7 / 14$ & $12 / 32$ \\
\hline \multicolumn{4}{|l|}{ Histology } \\
\hline Adenoca/others & $17 / 6$ & $15 / 6$ & $32 / 12$ \\
\hline \multicolumn{4}{|l|}{ Previous treatment } \\
\hline None & II & II & 22 \\
\hline $\mathrm{HPT}^{\mathrm{a}}$ & 6 & 8 & 14 \\
\hline Operation & 5 & 2 & 7 \\
\hline Radiotherapy & । & 0 & । \\
\hline \multicolumn{4}{|c|}{ aHypotonic cisplatin treatment. ECOG = Eastern Cooperative Oncology Group. } \\
\hline \multicolumn{4}{|c|}{ Table 2 Treatment delivery } \\
\hline Cycle of treatment & $\begin{array}{c}<75 \text { years } \\
(n=23)\end{array}$ & $\begin{array}{c}\geqslant 75 \text { years } \\
(n=21)\end{array}$ & $\begin{array}{c}\text { Total } \\
(n=44)\end{array}$ \\
\hline I & $23(100 \%)$ & $21(100 \%)$ & $44(100 \%)$ \\
\hline 2 & $21(91 \%)$ & $18(86 \%)$ & 39 (89\%) \\
\hline 3 & 12 (52\%) & 14 (67\%) & 26 (59\%) \\
\hline 4 & $9(39 \%)$ & II (52\%) & 20 (45\%) \\
\hline$\geqslant 5$ & $6(26 \%)$ & $10(48 \%)$ & $16(36 \%)$ \\
\hline \multicolumn{4}{|l|}{ No. of cycles } \\
\hline Median & 3 & 4 & 3 \\
\hline Range & $1-18$ & $1-16$ & $1-18$ \\
\hline
\end{tabular}

thrombocytopenia was observed. The frequency of grade 3 and 4 haematologic toxicities was $57 \%$ in the younger-age group and $62 \%$ in the older-age group. The frequency of grade 3 or greater nonhaematologic toxicities was $5 \%$ or less. No grade 4 nonhaematologic toxicity was observed.

\section{Response}

Among the 44 patients, 18 patients showed a partial response (41\%; 95\% confidence interval, 26-55\%). There were 24 patients (55\%) with no change and two patients $(5 \%)$ with progressive disease. There were no differences in the response rate according to age ( $<75 v s \geqslant 75$ years, 44 vs $38 \%$ ), gender (male $v s$ female, 46 vs 33\%), stage (III vs IV, 42 vs 41\%) and histology (adenocarcinoma $v s$ others, $44 v s 33 \%)$. Median duration of response was 6.9 months.

\section{Survival}

The overall median follow-up time for all patients was 38 months (range from 23 to 50 months). As shown in Figure 1, the median survival time of all 44 patients was 13.2 months, and the survival rates at 1 and 2 years were $59 \%$ (95\% confidence interval, $45-74 \%$ ) and $34 \%$ (95\% confidence interval, $20-48 \%$ ), respectively. There were no statistically significant differences in survival between the patients under 75 and those over 75 years $(P=0.4948)$. The median survival time was 13.2 and 13.3 months, respectively.
Table 3 Haematologic and nonhaematologic toxicities $(n=44)$

\begin{tabular}{|c|c|c|c|c|c|}
\hline \multirow[b]{2}{*}{ Toxicity } & \multicolumn{4}{|c|}{ Grade } & \multirow[b]{2}{*}{ Frequency of Grade 3 or 4 (\%) } \\
\hline & I & 2 & 3 & 4 & \\
\hline Leukopenia & 4 & 19 & 13 & I & 32 \\
\hline Neutropenia & 5 & 8 & 16 & 9 & 57 \\
\hline Anemia & 22 & 13 & 4 & 0 & 9 \\
\hline Thrombocytopenia & 15 & 9 & 8 & 0 & 18 \\
\hline GOT & 8 & I & 0 & 0 & \\
\hline GPT & 10 & I & 0 & 0 & \\
\hline Creatinine & 1 & 0 & 0 & 0 & \\
\hline Anorexia & 4 & । & 2 & 0 & 5 \\
\hline Vomiting & 2 & 2 & 0 & 0 & \\
\hline Diarrhoea & 0 & I & 2 & 0 & 5 \\
\hline AIP & 0 & 0 & । & 0 & 2 \\
\hline Febrile neutropenia & 0 & 0 & I & 0 & 2 \\
\hline
\end{tabular}

$\mathrm{GOT}=$ glutamic oxaloacetic transaminase; GPT = glutamic pyruvic transaminase $\mathrm{ALP}=$ alkaline phosphatase

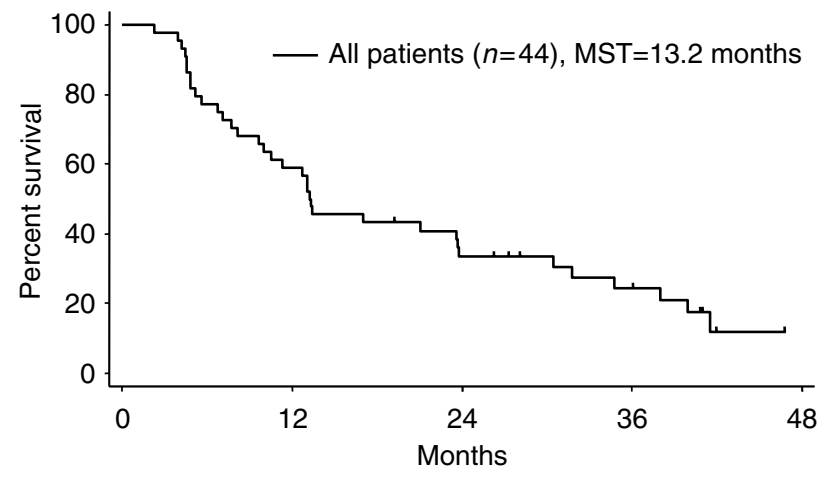

Figure I Overall survival. Each tick represents a patient who is alive.

\section{DISCUSSION}

Platinum-based combination chemotherapy is recommended for the treatment of advanced NSCLC patients with a good performance status (Pfister et al, 2004). However, the incidence of severe adverse effects induced by this combination chemotherapy is indeed more frequently observed in elderly patients than younger patients even if the subjects have good performance status (Langer et al, 2002). Therefore, a single agent such as vinorelbine, whose effect on prolonging survival in advanced NSCLC with elderly has been demonstrated in comparison to the best supportive care (Anonymous, 1999), is sometimes selected to treat such patients in practice. In addition, the combination of vinorelbine plus gemcitabine has been reported to be not more effective than single-agent vinorelbine or gemcitabine in the treatment of elderly patients with advanced NSCLC (Gridelli et al, 2003).

Lung cancer is primarily a disease of the elderly. More than one half of new diagnoses and more than two-thirds of annual deaths occur in patients over 65 years (Havlik et al, 1994; Gridelli et al, 1997). Although the aim of the present study was not to develop a treatment for only the elderly, the median age of the patients in this study was 74 years and $48 \%$ of the patients were 75 years or older. The reasons for this age distribution were thought to be partly due to the followings: First, the subjects of almost all previous clinical trials for chemotherapy against advanced NSCLC conducted in Japan were limited to patients under 75 years of age. Second, both UFT and gemcitabine were considered to be relatively safe anticancer agents. Third, they could be administered on an outpatient basis with no need for premedication and prehydration. 
The overall response rate of the present trial using UFT and gemcitabine was $41 \%$. In a phase I trial of this combination chemotherapy, the response rate of patients without prior chemotherapy was also reported to be $45 \%$ (Seto et al, 2002). This high antitumour effect may lend support to the sequence of administration of UFT and gemcitabine. In fact, an in vitro study has shown the sequence-dependent antitumour effects of the combination of 5-FU and gemcitabine to be seen with a maximum effect when 5FU preceded gemcitabine (Rauchwerger et al, 2000). Since the 5-FU concentration in blood reaches a steady state 5 days after the start of UFT administration (Ho et al, 1998), the administration of gemcitabine on days 8 and 15 is considered to be most appropriate.

Since the recent randomised trial demonstrated that gemcitabine plus carboplatin produced a significantly higher response rate and survival rate than gemcitabine alone in advanced NSCLC patients including a substantial proportion of elderly patients (37\% of patients $>70$ years old), the combination chemotherapy using gemcitabine and carboplatin (Sederholm, 2002) may also be considered to be a suitable chemotherapy regimen for the treatment of elderly patients. The response rate and median survival time in the combination chemotherapy group has been reported to be $30 \%$ and 10 months, respectively. Grade 3 or 4 nonhaematologic toxicity was observed in $26 \%$ of the patients, while grade 3 and 4 thrombocytopenia was reported in $24 \%$ each of patients, respectively. A toxicity profile in the elderly has not yet been reported.

In the present phase II trial of 44 patients with a median age of 74 years, including 21 patients who were 75 years or older, the response rate was $41 \%$ and the median survival time was 13.4 months. These observations suggest that this combination chemotherapy is also worthy of further investigation for the treatment of all NSCLC patients including the elderly. In addition, it should be confirmed whether or not this combination regimen is equally effective in ethnic groups other than Japanese.

\section{ACKNOWLEDGEMENTS}

We thank Mr Brian Quinn for his critical review and Ms Yumiko Oshima for her help in preparing the manuscript.

\section{REFERENCES}

Anonymous (1999) Effects of vinorelbine on quality of life and survival of elderly patients with advanced non-small-cell lung cancer. The Elderly Lung Cancer Vinorelbine Italian Study Group. J Natl Cancer Inst 91: 6672

Fujii S, Kitano S, Ikenaka K, Shirasaka T (1979) Effect of coadministration of uracil or cytosine on the anti-tumor activity of clinical doses of 1-(2tetrahydrofuryl)-5-fluorouracil and level of 5-fluorouracil in rodents. Gann 70: 209-214

Gridelli C, Perrone F, Gallo C, Cigolari S, Rossi A, Piantedosi F, Barbera S, Ferrau F, Piazza E, Rosetti F, Clerici M, Bertetto O, Robbiati SF, Frontini L, Sacco C, Castiglione F, Favaretto A, Novello S, Migliorino MR, Gasparini G, Galetta D, Iaffaioli RV, Gebbia V (2003) Chemotherapy for elderly patients with advanced non-small-cell lung cancer: the Multicenter Italian Lung Cancer in the Elderly Study (MILES) phase III randomized trial. J Natl Cancer Inst 95: 362-372

Gridelli C, Perrone F, Monfardini S (1997) Lung cancer in the elderly. Eur J Cancer 33: 2313-2314

Harper P (2003) Update on gemcitabine/carboplatin in patients with advanced non-small cell lung cancer. Semin Oncol 30: 2-12

Havlik RJ, Yancik R, Long S, Ries L, Edwards B (1994) The National Institute on Aging and the National Cancer Institute SEER collaborative study on comorbidity and early diagnosis of cancer in the elderly. Cancer 74: $2101-2106$

Ho DH, Pazdur R, Covington W, Brown N, Huo YY, Lassere Y, Kuritani J (1998) Comparison of 5-fluorouracil pharmacokinetics in patients receiving continuous 5 -fluorouracil infusion and oral uracil plus $\mathrm{N1}$ (2'-tetrahydrofuryl)-5-fluorouracil. Clin Cancer Res 4: 2085-2088

Huang P, Chubb S, Hertel LW, Grindey GB, Plunkett W (1991) Action of $2^{\prime}, 2^{\prime}$-difluorodeoxycytidine on DNA synthesis. Cancer Res 51: 6110-6117

Ichinose Y, Seto T, Yamamoto H, Ito K, Araki J, Ushijima S, Inoue Y, Semba H (2003) Intrapleural hypotonic cisplatin treatment for malignant pleural effusion in 80 patients with non-small cell lung cancer. In Proceedings of the American Society of Clinical Oncology, Deborah Whippen, (ed) Vol. 22, p 638. Chicago, USA: W.B. Saunders Company, (abstract 2566)

Ichinose Y, Takanashi N, Yano T, Asoh H, Yokoyama H, Tayama K, Hara N, Ohta M (1995) A phase II trial of oral tegafur and uracil plus cisplatin in patients with inoperable nonsmall cell lung cancer. Cancer 75: $2677-2680$

Ichinose Y, Yosimori K, Yoneda S, Kuba M, Kudoh S, Niitani H (2000) UFT plus cisplatin combination chemotherapy in the treatment of patients with advanced non-small cell lung carcinoma: a multiinstitutional phase II trial. For the Japan UFT Lung Cancer Study Group. Cancer 88: $318-323$
Kato H, Ichinose $\mathrm{Y}$, Ohta $\mathrm{M}$, Hata E, Tsubota N, Tada H, Watanabe $\mathrm{Y}$, Wada H, Tsuboi M, Hamajima N (2004) A randomized trial of adjuvant chemotherapy with uracil-tegafur for adenocarcinoma of the lung. $N$ Engl J Med 350: 1713-1721

Keicho N, Saijo N, Shinkai T, Eguchi K, Sasaki Y, Tamura T, Sakurai M, Sano T, Hoshi A (1986) Phase II study of UFT in patients with advanced non-small cell lung cancer. Jpn J Clin Oncol 16: 143-146

Langer CJ, Manola J, Bernardo P, Kugler JW, Bonomi P, Cella D, Johnson DH (2002) Cisplatin-based therapy for elderly patients with advanced non-small-cell lung cancer: implications of Eastern Cooperative Oncology Group 5592, a randomized trial. J Natl Cancer Inst 94: $173-181$

Madajewicz S, Petrelli N, Rustum YM, Campbell J, Herrera L, Mittelman A, Perry A, Creaven PJ (1984) Phase I-II trial of high-dose calcium leucovorin and 5-fluorouracil in advanced colorectal cancer. Cancer Res 44: $4667-4669$

Miller AB, Hoogstraten B, Staquet M, Winkler A (1981) Reporting results of cancer treatment. Cancer 47: 207-214

Peters GJ, van der Wilt CL, van Moorsel CJ, Kroep JR, Bergman AM, Ackland SP (2000) Basis for effective combination cancer chemotherapy with antimetabolites. Pharmacol Ther 87: 227-253

Pfister DG, Johnson DH, Azzoli CG, Sause W, Smith TJ, Baker Jr S, Olak J, Stover D, Strawn JR, Turrisi AT, Somerfield MR (2004) American Society of Clinical Oncology treatment of unresectable non-small-cell lung cancer guideline: update 2003. J Clin Oncol 22: 330-353

Rauchwerger DR, Firby PS, Hedley DW, Moore MJ (2000) Equilibrativesensitive nucleoside transporter and its role in gemcitabine sensitivity. Cancer Res 60: 6075-6079

Saito J, Nakai Y, Saijo Y, Nukiwa T, Koinumaru S, Matsuura Y, Aso N, Yamane Y, Tsukamoto T, Sayama T, Nakabayashi T (2001) A phase II trial of oral UFT plus cisplatin (CDDP) in patients with non-small cell lung cancer (NSCLC). Lung Cancer 31: 285-293

Schulz L, Schalhorn A, Wilmanns W, Heinemann V (1998) Synergisic interaction with $\mathrm{dFdC}$ and 5-FU in colon cancer cells. In Proceedings of the American Society of Clinical Oncology Vol. 17, pp 251. Los Angeles, USA: W.B. Saunders Company, (abstract 965)

Sederholm C (2002) Gemcitabine compared with gemcitabine plus carboplatin in advanced NSCLC: a phase III study by the Swedish Lung Cancer Study Group. In Proceedings of the American Society of Clinical Oncology, Deborah Whippen, (ed) Vol. 21, p 291. Orland, USA: Lippincott Williams \& Wilkins, (abstract 1162)

Seto T, Yoh K, Asoh H, Yamamoto H, Semba H, Ichinose Y (2002) A phase I study of combination chemotherapy with gemcitabine and oral UFT for advanced non-small cell lung cancer. Br J Cancer 86: 1701 - 1704 